\title{
Stray-field nuclear magnetic resonance imaging in microgravity conditions
}

\author{
Leoncio Garrido ${ }^{1, a)}$ and José Sampayo ${ }^{2}$ \\ ${ }^{1}$ Departamento de Química Física, Instituto de Ciencia y Tecnología de Polímeros, CSIC, Juan de la \\ Cierva 3, 28006 Madrid, Spain \\ ${ }^{2}$ Departamento de Anatomía, Embriología y Anatomía Patológica, Facultad de Veterinaria, Universidad \\ Complutense, Avda. Puerta de Hierro s/n, 28040 Madrid, Spain
}

(Received 19 October 2007; accepted 15 December 2007; published online 6 March 2008)

\begin{abstract}
Magnetic levitation has been proposed as an alternative approach to simulate on Earth microgravity conditions encountered in space, allowing the investigation of weightlessness on materials and biological systems. In general, very strong magnetic fields, $15 \mathrm{~T}$ or higher, are required to achieve levitation for a majority of diamagnetic substances. Here, we show that it is possible to achieve levitation of these substances in a commercial superconductive magnet operating with a nuclear magnetic resonance (NMR) spectrometer at $9.4 \mathrm{~T}$ at ambient conditions. Furthermore, stray-field proton NMR imaging is performed in situ at the location where a sample is levitating, showing that it is feasible to obtain the corresponding one-dimensional profile. Considering that water is a diamagnetic substance and the main constituent of living systems, the outlined approach could be useful to investigate alterations in water proton NMR properties induced by low gravity and magnetic forces upon levitating, e.g., seeds, cells, etc. In addition to protons, it would also be possible to observe other nuclei (e.g., ${ }^{19} \mathrm{~F},{ }^{31} \mathrm{P}$, etc.) that may be of interest in metabolic and therapeutic investigations. (C) 2008 American Institute of Physics. [DOI: 10.1063/1.2842406]
\end{abstract}

The proposal of manned missions to Mars and the renewed interest in the exploration of the Moon increases the awareness on the effect of microgravity upon human beings and, in general, living systems. It is known that prolonged stays of astronauts in space lead to muscle and bone mass loss and immunodepression. To achieve a better understanding of the cellular mechanisms involved in the biological response to weightlessness conditions and develop physical and chemical countermeasures, more research is needed. The performance of experiments in space, i.e., on board the International Space Station, is costly and very limited in number due to the human and environment requirements of space missions. In this context, magnetic levitation has been proposed as a ground based alternative to experimentation in space and to investigate the effect of microgravity on living and inanimate systems. ${ }^{1-6}$

Diamagnetic materials immersed in a gradient magnetic field experience a repulsive force away from the higher field region. Considering that water is the most significant diamagnetic substance present at high concentration in biological systems, levitation of cells, seeds, small plants, and animals has been achieved. Magnetic levitation is accomplished when the gravitational force on a substance, $s$, placed in a magnet is compensated by the buoyancy forces induced by the medium, $m$, and the magnetic field. In other words, when

$$
\left(\rho_{s}-\rho_{m}\right) g=\left[\left(\chi_{s}-\chi_{m}\right) / \mu_{0}\right] B_{z} d B_{z} / d z,
$$

where $\rho$ is the density, $g$ is the gravitational acceleration, $\chi$ is the magnetic susceptibility, $\mu_{0}$ is the permeability of vacuum, $B$ is the magnetic field, and $z$ is the position along the normal to Earth's surface. When the medium is air, the

\footnotetext{
${ }^{\text {a) }}$ Author to whom correspondence should be addressed. Electronic mail: lgarrido@cetef.csic.es.
}

associated buoyancy forces [approximately 4\% (Ref. 7)] can be neglected and to levitate water, the magnetic force, $B_{z}$ $d B_{z} / d z$, needed is about $1400 \mathrm{~T}^{2} \mathrm{~m}^{-1}$, which has been accomplished using very intense magnetic fields, in excess of $15 \mathrm{~T}$. Increasing the magnetic and gravitational buoyancy forces induced by the medium may lower this requirement. It has been shown that drops of water levitate in pressurized air and oxygen at 60 and 12 atm, respectively, using magnetic fields of about $10 \mathrm{~T}^{7}$ Also, dense samples of, i.e., gold, lead, and silicon have been levitated in liquid oxygen. ${ }^{8}$

The approach outlined here facilitates the levitation of a diamagnetic substance in an aqueous medium with a $9.4 \mathrm{~T}$ superconductive magnet at atmospheric pressure by doping water with a paramagnetic compound approved by the Food and Drug Administration of the United States for clinical use as magnetic resonance imaging (MRI) contrast agent [diethylene triamine penta-acetic acid Gd(III) (GdDTPA)]. Furthermore, one-dimensional (1D) proton stray-field MRI on levitating samples is accomplished using a quadrature echo pulse sequence consisting of $(\alpha)_{x}-\tau-(\alpha)_{y}-\tau$-echo and varying the spectrometer carrier frequency stepwise within a suitable range of the resonance frequency at the levitation position. Differences between the relaxation times of protons in the samples and medium are exploited to enhance image profile contrast.

The experiments were performed on a Bruker Avance 400 spectrometer (Bruker Analytik GmbH, Karlsruhe, Germany) equipped with a Bruker UltraShield 9.4 $\mathrm{T}$ (proton Larmor frequency of $400.14 \mathrm{MHz}$ ), $89 \mathrm{~mm}$ vertical bore superconductive magnet and using a homebuilt NMR probe with a saddle type of radio frequency ( $\mathrm{rf}$ ) coil, $3 \mathrm{~cm}$ in diameter and $3.5 \mathrm{~cm}$ in length, tunable to the range of frequencies required. The levitation of polystyrene beads [Fig. 1(a)] in an aqueous solution of GdDTPA, $[\mathrm{Gd}(\mathrm{III})]=10 \mathrm{mM}$, oc- 


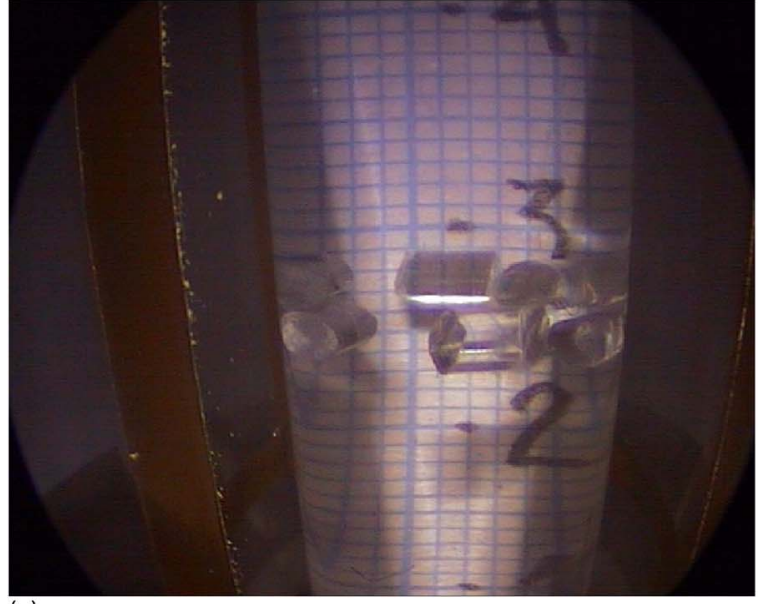

(a)

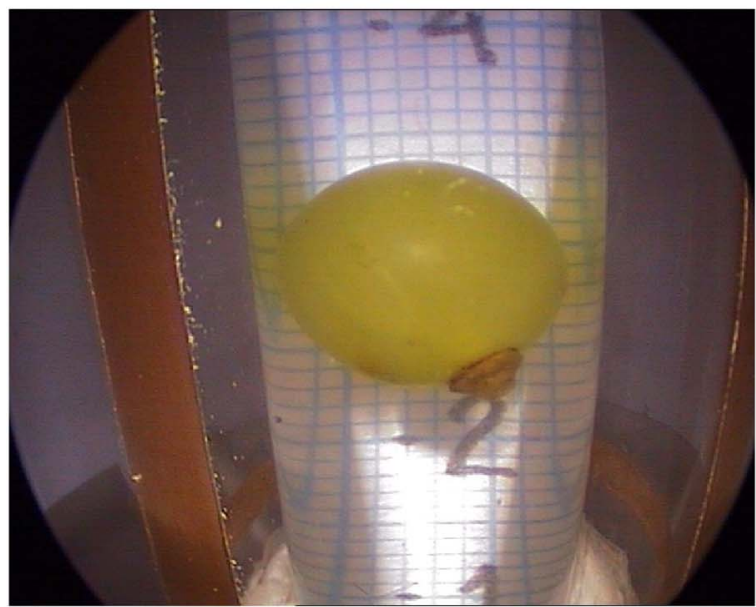

(b)

FIG. 1. (Color online) Photographs showing (a) polystyrene beads (bead size: $2 \times 3 \times 3 \mathrm{~mm}^{3}$, approximately) and (b) a grape with a diameter of $\sim 9 \mathrm{~mm}$, both samples levitated in aqueous solutions of the paramagnetic agent Gd(III)DTPA at concentrations of 10 and $12 \mathrm{mM}$, respectively. To aid visualization of sample size, a millimeter scale is shown.

curred at $26.7 \mathrm{~cm}$ above the center of the magnet, where the magnitude of the magnetic field was $3.26 \mathrm{~T}$ and the field gradient was $46.5 \mathrm{~T} / \mathrm{m}$ according to our measurements of the field with a Hall probe and gaussmeter (Lake Shore Cryotronics Inc., Westerville, $\mathrm{OH}$ ). The displacement of the beads along the $z$ axis from the bottom of a test tube to their final location was recorded using an endoscope (Karl Storz $\mathrm{GmbH}$, Tuttlingen, Germany) having a $70^{\circ}$ vision angle and a field of view of $67^{\circ}$ and equipped with Techno Pack X. Also, a grape [Fig. 1(b)] was levitated at about the same $z$ location using the experimental setup just described with a concentration of Gd(III)DTPA equal to $12 \mathrm{mM}$.

Stray-field NMR imaging ${ }^{9}$ was used to acquire a $1 D$ proton profile of the sample shown in Fig. 1(a). The NMR probe was tuned to the corresponding proton Larmor frequency at values ranging from -6 to $+10 \mathrm{MHz}$ with respect to the reference frequency of $136.6 \mathrm{MHz}$ at the center of the coil that was set to $0.0 \mathrm{~mm}$ (see Fig. 2). The presence of the beads within the field of view (FOV) defined between -6 and $+10 \mathrm{MHz}$ causes a decrease in signal intensity mainly due to differences between the relaxation times (spin-lattice $T_{1}$ and spin-spin $T_{2}$ ) of protons in the sample and the medium. The

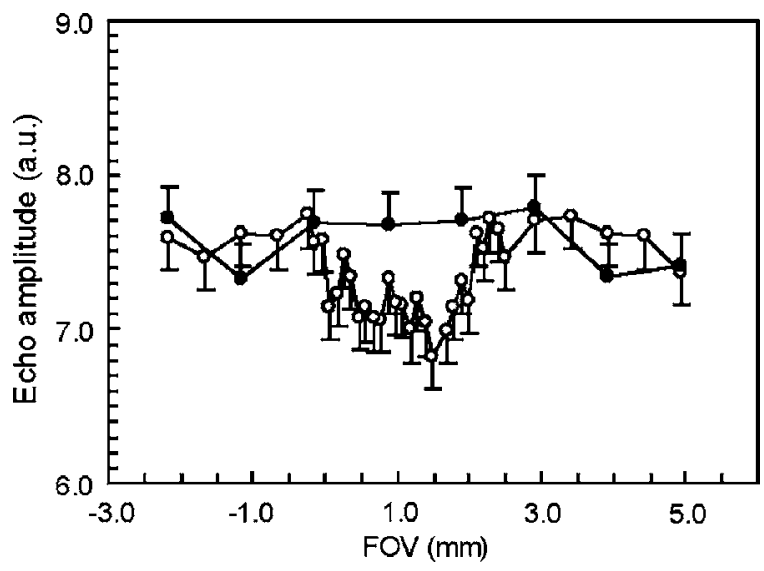

FIG. 2. $T_{1}$-weigthed proton NMR profile (open circles) corresponding to the sample shown in Fig. 1(a). The decrease in signal intensity in the region between 0 and $2.5 \mathrm{~mm}$ with respect to the center of the rf coil indicates the presence of the polystyrene beads within the FOV setup in the NMR imaging experiment. The sampling of the region of interest was performed by varying the spectrometer frequency stepwise between +10 and $-6 \mathrm{MHz}$ (or their equivalent in millimeters: +5 and -3 , respectively) with respect to frequency at the center of the coil (set to $0 \mathrm{~mm}$ ). The $1 \mathrm{D}$ profile represents the amplitude of the echo at each of the 36 imaging planes along the $z$ axis. The plane thickness was $15 \mu \mathrm{m}$ and the gap between planes was $100 \mu \mathrm{m}$ at the region where the beads were located and $500 \mu \mathrm{m}$ elsewhere. For each imaging plane, 256 scans were collected with a repetition rate of $200 \mathrm{~ms}$. Here, differences in relaxation times of protons in water and polystyrene beads are exploited to enhance image contrast. The base line (closed circles) corresponds to an aqueous medium without the beads sampled at intervals of $2 \mathrm{MHz}(1 \mathrm{~mm})$. The error bars correspond to the rms of the NMR signal

use of a rf pulse sequence with a fast repetition rate $\left(T_{1}\right.$ weighting) enhances the detection of protons with short $T_{1}$ (in this case, the medium) compared to those with a long one (the polystyrene). The closed circles show a proton profile corresponding to the aqueous medium with $[\mathrm{Gd}(\mathrm{III})]$ at $12 \mathrm{mM}$.

Figure 3 illustrates the contrast profile corresponding to a grape levitating, as shown in Fig. 1(b). The amplitude of

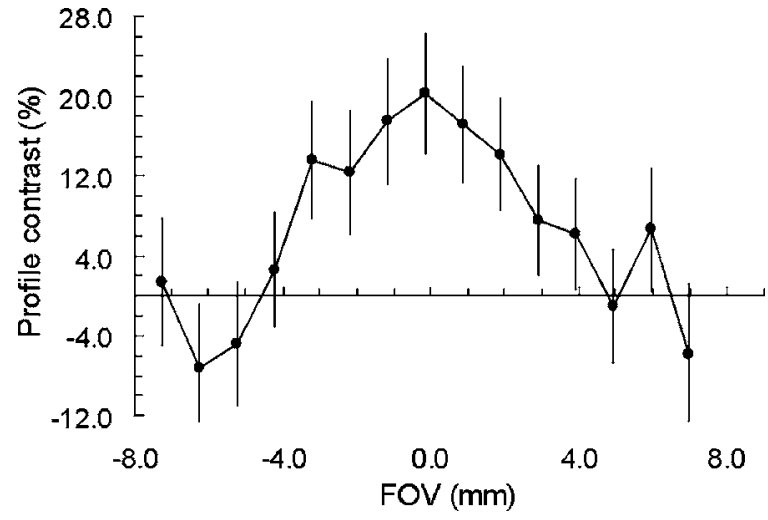

FIG. 3. Plot illustrating the profile contrast, $C(\%)$ $=\left(S_{\text {water }}-S_{\text {water }}\right.$ and grape $\left./ S_{\text {water }}\right) \times 100$, between the medium with the sample as shown in Fig. 1(b) and without the sample. For each acquisition, the sampling of the region of interest within the FOV is performed by varying the spectrometer frequency stepwise between $\pm 14 \mathrm{MHz}$ (or their equivalent in millimeters: \pm 7 ) with respect to the frequency at the center of the coil. The 1D profile represents the amplitude of the echo at each of the 15 regularly spaced imaging planes along the $z$ axis. The plane thickness is $15 \mu \mathrm{m}$ and the gap between planes is $1.0 \mathrm{~mm}$. For each imaging plane, 256 scans were collected with a repetition rate of $200 \mathrm{~ms}$. The rms of the NMR signal is used to calculate the standard deviation (bars) at each point. 
the profile at each location represents the normalized difference between the amplitude of the proton signal (echo) given by the medium alone and by the medium with the grape.

In addition to $T_{1}$, other mechanisms (i.e., molecular diffusion) could be used to exploit differences between specimens subject of study and enhance image contrast.

Here, proton imaging of levitating matter in a commercial 9.4 T NMR spectrometer is demonstrated. Gd(III)DTPA in a culture medium could be used to levitate free cells or cells seeded on a plastic substrate or embedded in polymer beads without compromising their viability since the osmotic pressure of mammalian cells is higher than that of the medium with the paramagnetic agent at the concentration used in this work. ${ }^{10}$ Moreover, Gd(III)DTPA is an extracellular contrast agent; it does not cross the cell membrane.

At the present, spectrometers with magnetic fields up to $21 \mathrm{~T}$ are available and, therefore, it may be possible to perform heteronuclear imaging on levitating systems in situ without a supporting medium. Compared to magnets commonly used in levitation research, the magnets in commercial NMR spectrometers offer higher field stability which could be advantageous in prolonged studies.

The sensitivity of NMR to chemical structure and molecular dynamics would enable the investigation of possible changes induced by microgravity and magnetic forces upon a variety of diamagnetic materials, living and inanimate. In addition to investigations using other techniques simulating weightlessness conditions (e.g., clinorotation, random positioning machine, etc.), control experiments to elucidate the effect of the magnetic force and its gradient could be performed along the $z$ direction inside the magnet, in the presence of a field gradient but at $g=2(-z)$ or in its absence at $g=1 \quad(z=0$, the center of the magnet). Furthermore, the approach outlined in this work could be used to investigate the effect of gravitational fields encountered on the Moon, as well as on Mars or on other planets, by placing the test specimen at the $z$ location where the balance between the magnetic forces and gravity matches that of interest. In the scenarios just indicated, at any time during the experiments, the sample could be placed briefly and repeatedly at the center of the magnet where the field is very homogeneous to acquire the corresponding high resolution NMR spectrum and to reposition it afterward. This maneuver could allow longitudinal monitoring of chemical or metabolic changes that may be induced in the test specimens by levitation and the presence of very strong magnetic field gradients.

The authors would like to thank T. Delfs (Karl Storz Endoscópica Ibérica S.A., Spain) for the loan of the endoscope equipment. Also, the technical assistance provided by S. Moñivas to build the NMR probe is acknowledged. This work was supported in part by the Consejo Superior de Investigaciones Científicas (CSIC).

${ }^{1}$ J. S. Brooks, J. A. Reavis, R. A. Medwood, T. F. Stalcup, M. W. Meisel, E. Steinberg, L. Arnowitz, C. C. Stover, and J. A. A. J. Perenboom, J. Appl. Phys. 87, 6194 (2000).

${ }^{2}$ M. D. Simon and A. K. Geim, J. Appl. Phys. 87, 6200 (2000).

${ }^{3}$ M. Motokawa, K. Watanabe, and S. Awaji, Curr. Appl. Phys. 3, 367 (2003).

${ }^{4}$ J. M. Valles, H. J. Maris, G. M. Seidel, J. Tang, and W. Yao, Adv. Space Res. 36, 114 (2005).

${ }^{5}$ E. Beaugnon and R. Tournier, Nature (London) 349, 470 (1991).

${ }^{6}$ K. Guevorkian and J. M. Valles, Appl. Phys. Lett. 84, 4863 (2004).

${ }^{7}$ Y. Ikezoe, N. Hirota, J. Nakagawa, and K. Kitazawa, Nature (London) 393, 749 (1998).

${ }^{8}$ A. T. Cathrall, L. Eaves, P. J. King, and S. R. Booth, Nature (London) 422, 579 (2003).

${ }^{9}$ P. J. McDonald, Prog. Nucl. Magn. Reson. Spectrosc. 30, 69 (1997).

${ }^{10}$ A. Winkleman, K. L. Gudiksen, D. Ryan, G. M. Whitesides, D. Greenfield, and M. Prentiss, Appl. Phys. Lett. 85, 2411 (2004). 